\title{
Effect of mild sepsis on behavioral and biochemical changes on the stress-induced animal model of depression
}

\author{
Clarissa M. Comim ${ }^{1 *}$, Napoleão C. Silva ${ }^{2}$, Paula Dias ${ }^{1}$, Bruna P. Mendonça² ${ }^{2}$, Gislaine Z. Réus ${ }^{2}$, Tatiana Barichello ${ }^{2-4}$, Felipe Dal-Pizzol ${ }^{5}$ and \\ João Quevedo $\mathbf{o}^{2-4,6}$
}

${ }^{1}$ Research Group in neurodevelopment of childhood and adolescence, Laboratory of Experimental Neuroscience, Postgraduate Program in Health Sciences, University of South Santa Catarina, Avenida Pedra Branca, 25 Pedra Branca, 88137-270 Palhoça, SC, Brazil

${ }^{2}$ Translational Psychiatry Laboratory, Graduate Program in Health Sciences, University of Southern Santa Catarina (UNESC), Criciúma, SC, Brazil

${ }^{3}$ Translational Psychiatry Program, Department of Psychiatry and Behavioral Sciences, McGovern Medical School, The University of Texas Health Science Center at Houston (UTHealth), Houston, TX, USA

${ }^{4}$ Neuroscience Graduate Program, The University of Texas MD Anderson Cancer Center UTHealth Graduate School of Biomedical Sciences, Houston, TX, USA ${ }^{5}$ Laboratory of Experimental Physiopathology, Graduate Program in Health Sciences, Health Sciences Unit, University of Southern Santa Catarina, Avenida Universitária, 1105, Pinheirinho, 88806-000 Criciúma, SC, Brazil

${ }^{6}$ Center of Excellence on Mood Disorders, Department of Psychiatry and Behavioral Sciences, McGovern Medical School, The University of Texas Health Science Center at Houston (UTHealth), Houston, TX, USA

\begin{abstract}
The systemic inflammation that occurs during severe sepsis can be the cause of long-term consequences. Studies have demonstrated a possible correlation between the neuroinflammatory process and depression. However, the effects of mild sepsis as a tolerant factor for some depressive parameters have not been described. Thus, the objective of this study is to evaluate the effect of mild sepsis on behavioral and biochemical changes on the stress-induced animal model of depression. To this aim, adult male Wistar rats were submitted to cecal ligation and perforation and after 30 days it was submitted to the chronic mild stress protocol (CMS). The animals were submitted to stressors for 40 days. To evaluate the efficacy of CMS induction anhedonia was determined as the amount of sweet food consumption. The levels of neurotrophins and oxidative damage were also evaluated in the hippocampus. It is observed that mild sepsis improved depressive-like parameters after CMS protocol associated with an increase of neurotrophins levels and a decrease of oxidative damage in the hippocampus. In conclusion, our data suggest that a previous mild systemic inflammation caused by the induction of mild sepsis could decrease consequences in the central nervous system (CNS) induced by CMS protocol.
\end{abstract}

\section{Introduction}

Severe sepsis often affects the brain. At admission, cerebral dysfunction may develop in up to $71 \%$ of septic patients. This condition is caused by systemic inflammation without brain infection and clinically characterized by mental processes impairment, disorientation, delirium or coma [1-3]. Clinical and pre-clinical researches have shown that sepsis is associated to the generation of pro- and anti-inflammatory cytokines [4-6], increase in the blood-brain barrier (BBB) permeability [4], impairment of cerebral microcirculation [7], oxidative stress [8], hippocampal apoptosis [9] long-term cognitive impairment [10,11] and depressive-like behavior [12]. However, studies have shown that prior mild sepsis has some modality-specific effects on the behavioral response to subsequent immune challenge [13-15].

In this context, some studies have associated the processes of neuroinflammation and depression [16-19]. Clinical studies have reported an increase in the inflammatory response during the course of major depression evidenced by an increase in pro-inflammatory cytokine levels in the serum [20,21]. Patients with major depression who are healthy seem to have activated inflammatory pathways, with an increase of pro-inflammatory cytokines, chemokines, acute-phase proteins and adhesion molecules [22-24]. Pro-inflammatory cytokines can enter and alter the brain function, even though they are released peripherally, modifying neurotransmitter metabolism, neuroendocrine function through hyperactivation of the hypothalamic-pituitaryadrenal (HPA) axis, neuronal plasticity, decrease of neurotrophin levels as BDNF and greater activation of the glutamatergic pathways, influencing the synaptic behaviors [25].

Studies demonstrate that a vulnerable brain can be more easily affected by responses associated with stress as a result of systemic inflammation [26-28]. In this context, some authors have shown that prior history of an immune challenge may affect central and behavioral responses to subsequent immune challenge, either leading to exaggerated responses via priming mechanisms or lessened responses via endotoxin tolerance [29-31]. Given the persistent upregulation of neuroinflammation following severe sepsis and that association between depression and neuroinflammation, we set out to enquire whether prior mild sepsis would impact on depressive-like parameters

${ }^{\star}$ Correspondence to: Clarissa M. Comim, Ph.D. Research group in neurodevelopment of childhood and adolescence, Laboratory of Experimental Neuroscience, University of South Santa Catarina, Palhoça, SC, Brazil, Tel: +55 48 32791167, E-mail: clarissamc@gmail.com

Key words: sepsis, inflammation, depression, stress, central nervous system

Received: August 05, 2020; Accepted: August 15, 2020; Published: August 21, 2020 
(anhedonia, oxidative damage and neurotrophins levels) induced by the chronic mild stress (CMS) response in the CNS. We used the chronic mild stress (CMS) paradigm as a model of depression and characterized its effects with to long-term outcomes after mild sepsis.

\section{Methods}

\section{Animals}

Male Wistar rats (60 days old, weighing 220-310g, $\mathrm{n}=50$ ) were obtained from the University of Southern Santa Catarina (UNESC) breeding colony. Animals were caged in groups of 5 with water and food ad libitum and were maintained on a $12 \mathrm{~h}$ light-dark cycle (lights on at 7:00 a.m.), at a temperature of $23^{\circ} \mathrm{C}$. All experimental procedures were performed by the approval of the Ethics Committee of Animals Use from UNESC (Protocol 051/2014) and conformed to international regulations.

\section{Mild sepsis induction}

Rats were subjected to cecal ligation and perforation (CLP) [3133]. Briefly, animals were anesthetized using a mixture of ketamine ( 80 $\mathrm{mg} / \mathrm{kg})$ and xylazine $(10 \mathrm{mg} / \mathrm{kg})$, given intraperitoneally. Under the aseptic condition, a $3-\mathrm{cm}$ midline laparotomy was performed to allow the exposure of the cecum with the adjoining intestine. The cecum was tightly ligated with a 3.0-silk suture at its base, below the ileocecal valve, and perforated once with a 20 -gauge needle. The cecum was then gently squeezed to extrude a small number of feces from the perforation site, returned to the peritoneal cavity, followed by the closure of the laparotomy with 4.0-silk sutures. The rats were volemically resuscitated with normal saline $(50 \mathrm{~mL} / \mathrm{kg}$ subcutaneously) immediately and $12 \mathrm{~h}$ after CLP. All animals were returned to their cages with free access to water and food. In the sham-operated group, the rats were submitted to all surgical procedures, but the cecum was neither ligated nor perforated. After surgery, both groups received $30 \mathrm{mg} / \mathrm{kg}$ ceftriaxone and $25 \mathrm{mg} / \mathrm{kg}$ clindamycin subcutaneously every $6 \mathrm{~h}$ for a total of 3 days. In the groups where the animals underwent sepsis, there was a $33 \%$ mortality. Survivors rats were divided in four experimental groups: 1) sham + control $(\mathrm{n}=10) ; 2)$ sham + CMS $(\mathrm{n}=10) ; 3)$ CLP + control $(\mathrm{n}=10)$ and 4$)$ CLP + CMS $(\mathrm{n}=10)$. After 30 days of induction, the CMS protocol was carried out as described below.

\section{Chronic mild stress protocol}

Chronic mild stress (CMS) protocol was adapted from [34]. Animals were divided in control $(n=20)$ and CMS $(n=20)$ groups. Controls were kept undisturbed in their home cages during the 40 days of the experiment, receiving only ordinary care with daily supports of food and water. The stressors used in this protocol were: (a) $24 \mathrm{~h}$ of water deprivation; (b) $24 \mathrm{~h}$ of food deprivation; (c) flashing light for $3 \mathrm{~h}$; (d) isolation (1-3 days); (e) $2 \mathrm{~h}$ of restraint and (f) $2 \mathrm{~h}$ of restraint at $4^{\circ} \mathrm{C}$. Animals were submitted to stressors for 40 days. Stress was applied at distinct periods every day and offered in a random order in to minimize its predictability.

To evaluate the efficacy of CMS induction anhedonia was determined as the amount of sweet food consumption. Briefly, rats were placed in a box $(40 \times 15 \times 20 \mathrm{~cm})$ divided into 9 equal rectangles. Ten Froot Loop(s) (Kellogg's $s^{\oplus}$ ) were placed in one extremity of the box. In the training trials, rats were exposed to the environment for $3 \mathrm{~min}$, once a day, during 5 consecutive days to become familiarized with the food. After, animals were exposed to 2 test sessions $(3 \mathrm{~min}$ each, on consecutive days) during which the number of ingested Froot Loop(s) was measured. Anhedonia test took 7-days in total and it was realized after the finish to the CMS $[34,35]$. Immediately after the behavioral tests, the rats were sacrificed by decapitation and the hippocampus dissected and stored at $80^{\circ} \mathrm{C}$.

\section{Measurement of neurotrophins levels}

For analysis of the neurotrophic factors, the total hippocampus were homogenized in phosphate-buffered solution (PBS) with $1 \mathrm{mM}$ phenylmethylsulfonyl fluoride (PMSF) and 1mM ethylene glycol bis(2aminoethylether)-N,N,N'N'-tetraaceticacid (EGTA). Homogenates were centrifuged at $10,000 \mathrm{~g}$ for $20 \mathrm{~min}$. Then, supernatants were collected for quantification of neurotrophic factors levels. BDNF, NGF and GDNF levels in the hippocampus were measured by sandwich enzyme-linked immunosorbent assay using commercial kits according to the manufacturer's instructions (NGF and BDNF levels were assessed with a kit from Chemicon (USA) and GDNF levels was assessed with a kit from Biosensis (USA)). Total protein was measured by the BCA method using bovine serum albumin as a standard.

\section{Oxidative damage}

Oxidative damage in lipids was assessed by measuring the formation of thiobarbituric acid reactive species (TBARS) during an acid-heating reaction, as previously described by Draper and Hadley (1990). Hippocampus samples were mixed with $1 \mathrm{~mL}$ of trichloroacetic acid $10 \%$ and $1 \mathrm{~mL}$ of thiobarbituric acid $0.67 \%$ and then heated for $30 \mathrm{~min}$. TBARS levels were determined spectrophotometrically by measuring the absorbance at $532 \mathrm{~nm}$. The Protein carbonyl was measured through determination of carbonyl groups content based on the reaction with dinitrophenylhidrazine (DNPH), as previously described by [35] Proteins were precipitated by the addition of $20 \%$ trichloroacetic acid and were redissolved in DNPH. The absorbance was monitored spectrophotometrically at $370 \mathrm{~nm}$. Protein content was measured by the BCA method using bovine serum albumin as a standard.

\section{Statistical analysis}

The Statistical Package for the Social Sciences (SPSS) 18.0 and GraphPad Prism version 6.0 were utilized for statistical analyses. Data from brain oxidative damage, neurotrophins and anhedonia were expressed as mean and standard derivation, and analyzed by factorial analysis of variance (ANOVA), followed by Tukey post-hoc test. For all comparisons, $\mathrm{p}<0.05$ was considered statistically significant.

\section{Results}

The parameters of anhedonia are shown in Figure 1. The animals of sham + CMS and CLP + control group demonstrated significantly decreased in Froots Loops intake when compared to the animals in the Sham + Control group. The animals of the CLP + Control group showed a decrease of Froots Loops intake when compared to Sham + Control animals. However, there was a significant difference in food intake between CLP + CMS and CLP + control groups, demonstrating possible protection.

Figure 2 shows the levels of neurotrophins through analysis of BDNF (Figure 2A), NGF (Figure 2B) and GDNF (Figure 2C) in the hippocampus. Animals of sham + CMS and CLP + control groups showed a significant decrease in the BDNF, NGF e GDNF hippocampus levels when compared to the sham + control group. However, the animals of the CLP + CMS group showed an increase of BDNF, NGF e GDNF hippocampus levels when compared to CLP + control animals.

Figure 3 demonstrates the results of oxidative damage in the hippocampus evaluating the protein carbonylation (Figure 3A) 


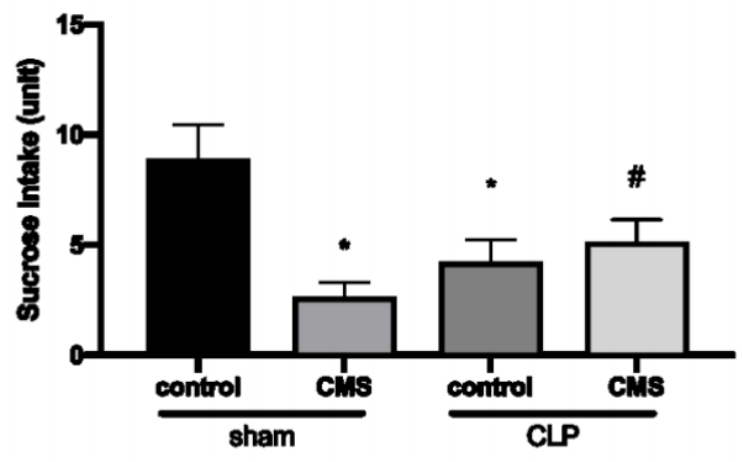

Figure 1. Anhedonia test. The data is expressed by mean and standard derivation. ${ }^{*} \mathrm{p}<0.05$ versus sham + control group; $\# \mathrm{p}<0.05$ versus CLP + control

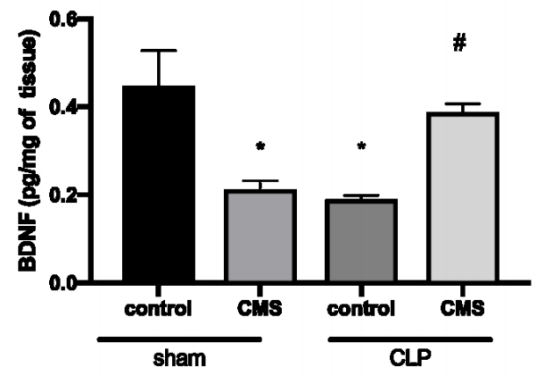

A

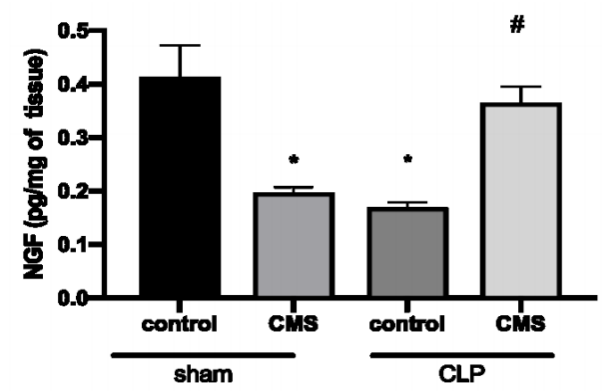

B

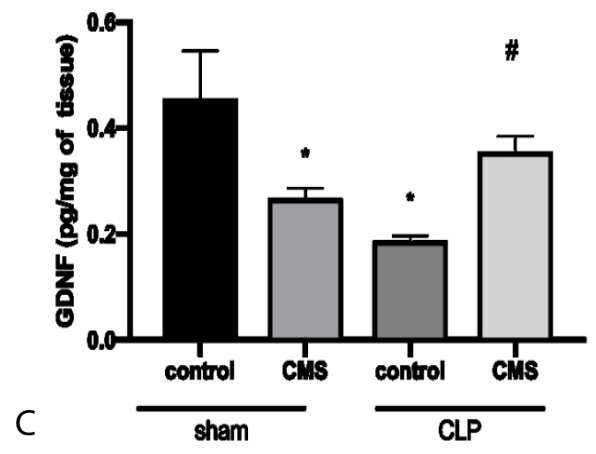

Figure 2. Neurotrophin levels in the hippocampus through evaluation of BDNF ( $2 \mathrm{~A})$, NGF (2B) and GDNF (3C). The data is expressed by mean and standard derivation. * $\mathrm{p}<0.05$ versus sham + control group; \#p $<0.05$ versus CLP + control
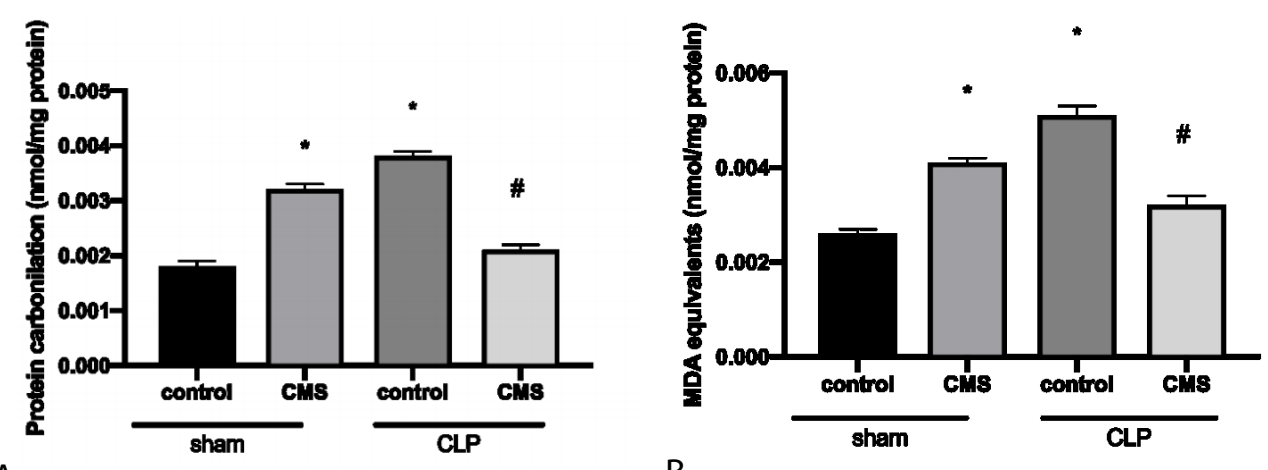

A

Figure 3. Oxidative damage in the hippocampus through the evaluation of protein carbonylation ( $3 \mathrm{~A})$ and lipidic peroxidation (3B). The data is expressed by mean and standard derivation. $* \mathrm{p}<0.05$ versus sham + control group; $\# \mathrm{p}<0.05$ versus CLP + control 
and lipid peroxidation (Figure 3B). There was an increase of protein carbonylation and lipid peroxidation in the hippocampus of the sham + CMS and the CLP + control groups when compared to the sham + control group. The animals the of CLP + CMS group showed a decrease of protein carbonylation and lipid peroxidation when compared to CLP + control animals.

\section{Discussion}

This study demonstrates that mild sepsis improved depressive-like behavior assessed by the anhedonia test after CMS protocol and this was related to an increase of the neurotrophins levels and a decrease in the protein carbonylation and lipid peroxidation in the hippocampus.

Studies have shown that oxidative stress can be associated with the sepsis immune alterations and that inhibitory methods to treat sepsis consequences can be a new study pathway of the possible resolution. In this context, endotoxin tolerance has been shown to develop an immune response that is protective of sepsis. After the tolerance induction, the organism becomes resistant [36-38] and this tolerance represents an immune adaptation response to a first stimulus that cannot be solved by the innate response [39]. The mechanisms of tolerance have been associated with protection against tissue damages in the hepatic and renal ischemia/reperfusion [40,41] and hemorrhagic shock [42] in animal models. In these studies, there was increased resistance to stressful situations showing that tolerance is far more than altered inflammatory responsiveness.

During the development of sepsis, it is generally believed that excessive inflammation leads to organ dysfunction. In this study, mild sepsis per se causes a depressive-like behavior characterized by the decrease of sucrose intake associated with the increase of oxidative damage and the decrease of neurotrophins. However, the depressive-like animal model caused by CMS protocol is also associated with increased oxidative damage [43] and a decreased of neurotrophins levels [44]. In other inflammatory diseases, such as meningitis it can be observed the correlation between cognitive impairment and low levels of BDNF [45]. The animals submitted to mild sepsis and exposed to CMS protocol after 30 days did not show depressive-like behavior, oxidative damage or altered levels of neurotrophins in the hippocampus. It is possible that this protection is due to tolerance caused by previous sepsis and prepare an animal to deal with further stressors such as CMS protocol.

In a recent study, it was demonstrated that the previous stress caused by CMS can protect the brain against the systemic acute and severe stress elicited by sepsis [46]. In this study we found the opposite: that mild sepsis can be associated with a possible modulation of neurotrophin levels and oxidative damage in the hippocampus. This modulation can be related to the prevention of depressive-like behavior after the CMS protocol. It is known that major depression is associated with worse outcomes in critically ill patients [47-49]. However, this outcome was not evaluated in this study.

It is important to note some limitations of this study [50,51]. We used a model of mild sepsis, which has provided clinically relevant information. However, it was not possible to determine if there is a "dose-dependent" effect. In a future study, other severity models should be used to detect more accurately a possible tolerance. Another important point is that this study did not fully explore a mechanism to elucidate the modulatory effects of systemic inflammation. It has however provided some evidence that this must include mechanisms associated with the control of oxidative stress and neurotrophins, but not inflammation [52]. Thus, it seems clear that somehow systemic inflammation related to mild sepsis modulates the brain response after stress promoted by the CMS protocol, and this may have an impact on the long-term brain dysfunction [53-55]. In conclusion, our data suggest that a previous systemic inflammation caused by the induction of mild sepsis could decrease the long-term depressive-like behavior and hippocampal oxidative damage associated with an increase of neurotrophin levels induced by CMS protocol.

\section{Conflict of interest}

The authors declare that they have no conflict of interest.

\section{Acknowledgments}

This research was supported by grants from University of South Santa Catarina (CMC), University of Southern Santa Catarina (TB, FDP and JQ) and National Council for Scientific and Technological Development (CMC, TB, FDP and JQ). CMC, TB, FDP and JQ is National Council for Scientific and Technological Development Research Fellows.

\section{Ethical approval}

All applicable international, national, and/or institutional guidelines for the care and use of animals were followed. All procedures performed in studies involving animals were in accordance with the ethical standards of the institution or practice at which the studies were conducted. Ethics Committee of Animals Use - UNESC 051/2014.

\section{References}

1. Wilson JX, Young GB (2003) Progress in clinical neurosciences: sepsis-associated encephalopathy: evolving concepts. Can J Neurol Sci 30: 98-05. [Crossref]

2. Semmler A, Hermann S, Mormann F, Weberpals M (2008) Sepsis causes neuroinflammation and concomitant decrease of cerebral metabolism. $J$ Neuroinflammation 15: 5-38.

3. Widmann CN, Heneka MT (2014) Long-term cerebral consequences of sepsis. Lancet Neurol 13: 630-636.

4. Comim CM, Cassol-Jr OJ, Constantino LS, Felisberto F, Petronilho F, et al. (2011a) Alterations in inflammatory mediators, oxidative stress parameters and energetic metabolism in the brain of sepsis survivor rats. Neurochem Res 36: 304-311.

5. Comim CM, Vilela MC, Constantino LS, Petronilho F, Vuolo F (2011b) Traffic of leukocytes and cytokine up-regulation in the central nervous system in sepsis. Intensive Care Med 37: 711-718.

6. Ritter C, Tomasi CD, Dal-Pizzol F, Pinto BB, Dyson A (2014) Inflammation biomarkers and delirium in critically ill patients. Crit Care 23: 106.

7. Taccone FS, Su F, Pierrakos C, He X, James S, (2010) Cerebral microcirculation is impaired during sepsis: an experimental study. Crit Care 14: 28.

8. Barichello T, Fortunato JJ, Vitali AM, Feier G, Reinke A (2006) Oxidative variables in the rat brain after sepsis induced by cecal liga- tion and perforation. Crit Care Med 34: 886-889.

9. Comim CM, Barichello T, Grandgirard D, Dal-Pizzol F, Quevedo J (2013) Caspase-3 mediates in part hippocampal apoptosis in sepsis. Mol Neurobiol 47: 394-398.

10. Tuon L, Comim CM, Petronilho F, Barichello T, Izquierdo I (2008) Time-dependent behavioral recovery after sepsis in rats. Intensive Care Med 34: 1724-31.

11. Iwashyna TJ, Ely EW, Smith DM, Langa KM (2010) Long-term cognitive impairmen and functional disability among survivors of severe sepsis. JAMA 304: 1787-1794.

12. Comim CM, Cassol-Jr OJ, Constantino LC, Petronilho F, Constantino LS (2010) Depressive-like parame- ters in sepsis survivor rats. Neurotox Res 17: 279-286.

13. Spencer SJ, Field E, Pittman QJ (2010) Neonatal programming by neuroimmune challenge: effects on responses and tolerance to septic doses of lipopolysaccharide in adult male and female rats. J Neuroendocrinol 22: 272-281.

14. Anderson ST, O'Callaghan EK, Commins S, Coogan AN.

15. Zhang Z, Ji M, Liao Y, Yang J, Gao J (2018) Endotoxin tolerance induced by lipopolysaccharide preconditioning protects against surgery-induced cognitive impairment in aging mice. Mol Med Rep 17:3845-3852. 
16. Smith RS (1991) The macrophage theory of depression. Med Hypotheses 35: 298-306.

17. Howren MB, Lamkin DM, Suls J (2009) Associations of depression with C-reactive protein, IL-1, and IL-6: a meta-analysis. Psychosom Med 71: 171-186.

18. Kohler O, Krogh J, Mors O, Benros ME (2016) Inflammation in depression and the potential for anti-inflammatory treatment. Curr Neuropharmacol 14: 732-42. [Crossref]

19. Culmsee C, Michels S, Scheu S, Arolt V, Dannlowski U (2019) Mitochondria, Microglia, and the Immune System-How Are They Linked in Affective Disorders? Front Psychiatry 9: 9-739.

20. Jeon SW, Kim YK (2016) Neuroinflammation and cytokine abnormality in major depression: Cause or consequence in that illness? World J Psychiatry 6: 283-293.

21. Valkanova V, Ebmeier KP, Allan CL (2013) CRP, IL-6 and depression: a systematic review and meta-analysis of longitudinal studies. J Affect Disord 25: 736-44.

22. Raison CL, Capuron L, Miller AH (2006) Cytokines sing the blues: inflammation and the pathogenesis of depression. Trends Immunol 27: 24-31.

23. Yang K, Xie G, Zhang Z, Wang C, Li W (2007) Levels of serum interleukin (IL)-6, IL1 beta, tumour necrosis factor-alpha and leptin and their correlation in depression. Aust N Z J Psychiatry 41: 266-73.

24. Kim YK, Lee SW, Kim SH, Shim SH, Han SW (2007) Differences in cytokines between non-suicidal patients and suicidal patients in major depression. Prog Neuropsychopharmacol Biol Psychiatry 32: 356-61.

25. Carvalho LA, Bergink V, Wijkhuijs J, Hoogendijk JW, Birkenhager KT (2014) Inflammatory activation is associated with a reduced glucocorticoid receptor alpha/ beta expression ratio in monocytes of inpatients with melancholic major depressive disorder. Transl Psychiatry 4: 344.

26. Dantzer R (2009) Cytokine, Sickness Behavior, and Depression. Immunology and Allergy Clinics of North America 29: 247-264. [Crossref]

27. Hennessy E, Griffin EW, Cunningham C (2015) Astrocytes are primed by chronic neurodegeneration to produce exaggerated chemokine and cell infiltration responses to acute stimulation with the cytokines IL-1 $\beta$ and TNF- $\alpha$. Journal of Neuroscience 35 : $8411-8422$.

28. Lekander M, Karshikoff B, Johansson E, Soop A, Fransson P (2016) Intrinsic functional connectivity of insular cortex and symptoms of sickness during acute experimental inflammation. Brain, Behavior, and Immunity 56: 34-41.

29. McCusker RH, Kelley KW (2013) Immune-neural connections: how the immune system's response to infectious agents influences behaviour. $J$ Exp Biol 216: 84-98.

30. Griffin EW, Skelly DT, Murray CL, Cunningham C (2013) Cyclooxygenase-1dependent prostaglandins mediate susceptibility to systemic inflammation-induced acute cognitive dysfunction. $J$ Neurosci 33: 15248-15258.

31. Fink MP, Heard SO (1990) Laboratory models of sepsis and septic shock. Journal of Surgical Research 49: 186-196.

32. Schwalm MT, Pasquali M, Miguel SP, Dos Santos JP, Vuolo F (2014) Acute brain inflammation and oxidative damage are related to long-term cognitive deficits and markers of neurodegeneration in sepsis-survivor rats. Mol Neurobiol 49: 380-5.

33. Petronilho F, Périco SR, Vuolo F, Mina F, Constantino L (2012) Protective effects of guanosine against sepsis-induced damage in rat brain and cognitive impairment. Brain Behav Immun 26: 904-10.

34. Gamaro GD, Manoli LP, Torres ILS, Silveira R, Dalmaz C (2003) Effects of chronic variate stress on feeding behavior and on monoamine levels in different rat brain structures. Neurochemistry International 42:107-114. [Crossref]

35. Levine RL, Williams AJ, Stadtman EP, Shacter P (1994) Carbonyl assays for determination of oxidatively modified proteins. Methods in Enzymology 233: 346-357.
36. Foster SL, Hargreaves DC, Medzhitov R (2007) Gene-specific control of inflammation by TLR-induced chromatin modifications. Nature 447: 972-978.

37. Melo ES, Barbeiro HV, Ariga S, Goloubkova T, Curi R (2010a) Immune cells and oxidative stress in the endotoxin tolerance mouse model. Braz J Med Biol Res 43: 57-67. [Crossref]

38. Medzhitov R, Schneider DS, Soares MP (2012) Disease tolerance as a defense strategy Science 335: 936-941.

39. Melo ES, Barbeiro DF, Gorjão R, Rios EC, Vasconcelos D (2010b) Gene expression reprogramming protects macrophage from septic-induced cell death. Mol Immunol 47: 2587-2593.

40. Colletti LM, Remick DG, Campbell DA (1994) LPS pretreatment protects from hepatic ischemia/reperfusion. $J$ Surg Res 57: 337-343.

41. Heemann U, Szabo A, Hamar P, Müller V, Witzke O (2000) Lipopolysaccharide pretreatment protects from renal ischemia/ reperfusion injury. Possible connection to an interleukin-6 dependent pathway. Am J Pathol 156: 287-293.

42. Ackerman M, Reuter M, Flohé S, Bahrami S, Redl H (2001) Cytokine synthesis in the liver of endotoxin-tolerant and normal rats during hemorrhagic shock. $J$ Endotoxin Res 7: $105-112$.

43. Lucca G, Comim CM, Valvassori SS, Réus GZ, Vuolo F (2009) Effects of chronic mild stress on the oxidative parameters in the rat brain. Neurochem Int 54: 358-62.

44. Stepanichev M, Dygalo NN, Grigoryan G, Shishkina GT, Gulyaeva N (2014) Rodent models of depression: neurotrophic and neuroinflammatory biomarkers. Biomed Res Int 2014: 932757.

45. Barichello T, Belarmino E Jr, Comim CM, Cipriano AL, Generoso JS (2010) Correlation between behavioral deficits and decreased brain-derived neurotrophic [correction of neurotrofic] factor in neonatal meningitis. $J$ Neuroimmunol 223: 73-76.

46. Steckert AV, Dominguini D, Michels M, Abelaira HM, Tomaz DB (2017) The impact of chronic mild stress on long-term depressive behavior in rats which have survived sepsis. J Psychiatr Res 9: 47-53. [Crossref]

47. Xiong GL, Wei J, Robert C, Linda KS, Smith PK (2006) Prognosis of patients taking selective serotonin reuptake inhibitors before coronary artery bypass grafting. The American Journal of Cardiology 98: 42-47.

48. Foss-Nieradko B, Stepnowska M, Piotrowicz R (2012) Effect of the dynamics of depression symptoms on outcomes after coronary artery bypass grafting. Kardiologia Polska 70: 591-597.

49. Stenman M, Sartipy U (2018) Depression screening in cardiac surgery patients. Heart Lung Circ 16: 30488.

50. Comim CM, Constantino LC, Barichello T, Streck EL, Quevedo J (2009) Cognitive impairment in the septic brain. Curr Neurovasc Res 6: 194-203.

51. Frey BN, Valvassori SS, Gomes KM, Martins MR, Dal-Pizzol F (2006) Increased oxidative stress in submitochondrial particles after chronic amphetamine exposur. Brain Research 1097: 224-229.

52. He W, Fong Y, Marano MA, Gershenwald JE, Yurt RW (1992) Tolerance to endotoxin prevents mortality in infected thermal injury: association with attenuated cytokine responses. J Infect Dis 165: 859-864.

53. Hill MN, Kim GC, Hellemans PV, Gorzalka BB, Weinberg J (2012) Neurobiology of chronic mild stress: Parallels to major depression. Neuroscience \& Biobehavioral Reviews 36: 2085-2117.

54. Robba C, Crippa IA, Taccone FS (2018) Septic Encephalopathy. Curr Neurol Neurosci Rep 2: 82 .

55. Windmann S, Hill H (2014) Dissociating electrophysiological correlates of subjective, objective, and correct memory in investigating the emotion-induced recognition bias. Conscious Cogn 29: 199-211.

Copyright: $₫ 2020$ Comim CM. This is an open-access article distributed under the terms of the Creative Commons Attribution License, which permits unrestricted use, distribution, and reproduction in any medium, provided the original author and source are credited. 